\title{
El Pensamiento Probabilístico de los Profesores de Biología en Formación
}

\author{
Probabilistic Thinking of Biology Teachers in Training
}

\author{
Amable Moreno* \\ José María Cardeñoso ** \\ Francisco González-García ${ }^{* * *}$
}

\begin{abstract}
Resumen
Los futuros profesores de secundaria requieren de una formación acorde a las demandas de la sociedad del siglo XXI. Ello impone el desarrollo de un pensamiento que le permita interpretar y abordar los fenómenos de naturaleza aleatoria. El objetivo de este trabajo es determinar las tendencias de pensamiento probabilístico de los estudiantes de los profesorados de Biología de la provincia de Mendoza, Argentina. Para ello se aplicó un cuestionario a los 325 estudiantes que cursan esta carrera. El mismo consta de tres partes; la primera trata sobre las variables demográficas de los estudiantes, la segunda corresponde al reconocimiento de la aleatoriedad; y la tercera a la estimación de la probabilidad de diferentes sucesos. Las respuestas se analizaron a partir de la aplicación de diferentes técnicas estadísticas; el test de independencia, el análisis de la varianza, el test de Tukey, análisis de clusters y análisis discriminante. En primer lugar, se encontraron diferencias significativas entre el reconocimiento de la aleatoriedad y el contexto del suceso, siendo mayor en el contexto de juego que en el físico natural. Mientras que, en el contexto físico natural se afirma la aleatoriedad desde la causalidad, en el de juego se afirma desde la incertidumbre. En segundo lugar, no se encontró relación de dependencia entre el reconocimiento de la aleatoriedad y la edad de los estudiantes, como así tampoco con el nivel académico de los mismos. Respecto a la estimación de la probabilidad, los estudiantes argumentan fundamentalmente desde la equiprobabilidad y desde la contingencia. El análisis de clusters y análisis discriminante permitieron encontrar cuatro tendencias de pensamiento: incertidumbre, determinista, contingente y personalista.
\end{abstract}

Palabras-clave: Estudiantes. Biología. Tendencias. Pensamiento. Probabilístico.

\begin{abstract}
Prospective secondary teachers need training in accordance with the demands of the 21 st century. This requires the development of a thought that allows interpreting and addressing the phenomena of random nature. The aim of this work is to determine trends of probabilistic thinking of biology teachers in training from the province of Mendoza, Argentina. We applied a questionnaire to 325 students pursuing this career. It consists of three parts:

\footnotetext{
* Doctora en Enseñanza de las Ciencias y la Tecnología en la Univ. Granada, Granada, España. Dirección postal: Liniers n.575, 5501, Godoy Cruz, Mendoza, Argentina. E-mail: amoreno@ fce.uncu.edu.ar.

** Doctor en Filosofía y Ciencias de la Educación: Ciencias de la Educación, por la Univ. Cádiz. Titular Universidad del Área de Didáctica de la Matemática, Dto. Didáctica. Univ. Cádiz, Cádiz, España. Dirección postal: Av. Constitución de 1978, n.6, 4º C, Urb. "La Noria", 11100, San Fernando, Cádiz, España E-mail: josemaria.cardenoso@uca.es.

*** Doctor en Ciencias Biológicas por Univ. Granada. Titular Universidad del Área de Didáctica de las Ciencias Experimentales. Director de Departamento Didáctica de las Ciencias Experimentales, Granada, España. Dirección postal: Facultad de Educación. Campus de Cartuja, 18071, Granada, España. E-mail: pagoga@ugr.es.
} 
the first deals with the demographic variables of students, the second is the recognition of randomness, and the third is the estimation of the probability of events. Responses were analyzed from the application of different statistical techniques; the independence test, analysis of variance, Tukey's test, cluster analysis and discriminant analysis were used. Significant differences between the recognition of randomness and the context of the event were found, being greater in the context of the game rather than the physical natural environment. Meanwhile, in the physical natural context states randomness is stated from causality, in gambling it states from uncertainty. Besides there was no relationship of dependency between the recognition of randomness and the age of the students, as well neither with their academic level. Regarding the estimation of probability, students argue mainly from the equiprobability and from contingency. Cluster analysis and discriminant analysis allowed us to find four trends of thought: uncertainty, causality, contingent and personalistic.

Keywords: Students. Biology. Trends. Thinking. Probabilistic.

\section{Introducción}

La alfabetización estadística es necesaria en la formación profesional de los futuros profesores de secundaria, para desarrollar la capacidad de analizar, interpretar y comunicar la información a partir de los datos extraídos de las situaciones del entorno (AZCÁRATE; CARDEÑOSO, 2011). Lo que implica el desarrollo de un pensamiento que incluya el reconocimiento de la incertidumbre presente en fenómenos de la vida real, para la toma de decisiones adecuadas (MORENO; CARDEÑOSO; GONZÁLEZ, 2010).

Desde hace más de quince años que se viene expresada esta necesidad; así (BOROVNICK; PEARD, 1996, p.240), afirman: "hay dos razones principales para legitimar la probabilidad en la currícula de cualquier nivel. Una es la formación de una forma específica de pensar, un pensamiento probabilístico. La segunda es la necesidad de su conocimiento para múltiples aplicaciones”. Posteriormente, en el The 18 th ICMI Study de 2008, Borovnick plantea los argumentos que justifican el rol de la probabilidad en la currícula estocástica: (a) la probabilidad es indispensable para la comprensión de los métodos de la Estadística Inferencial; (b) la probabilidad ofrece una herramienta para la modelización y/o creación de la realidad; (c) la probabilidad ofrece un tipo de pensamiento con el que se puede reflexionar sobre la realidad.

En este trabajo nos proponemos conocer las concepciones de los profesores en formación sobre la aleatoriedad y la estimación de la probabilidad. Esta información es de fundamental importancia para afrontar la enseñanza de la probabilidad en el Profesorado de Biología con estrategias didácticamente apropiadas; para desarrollar en los estudiantes intuiciones estables sobre la probabilidad y que les permita percatarse del potencial que encierra la misma (BOROVNICK, 2011). Sin dejar de considerar la importancia que tienen en la construcción de otros conceptos fundamentales, como es el de variable aleatoria y 
distribución de probabilidad de una variable aleatoria (RUIZ; BATANERO; ARTEAGA 2011).

\section{Investigaciones previas}

A partir del siglo XX los psicólogos han llevado a cabo una amplia investigación sobre la capacidad de las personas de producir o percibir la aleatoriedad. En el primer caso, los sujetos han tenido que simular resultados de un proceso aleatorio, como lanzar una moneda (WAGENAAR, 1972); en el segundo caso, los sujetos han tenido que evaluar la aleatoriedad de secuencias obtenidas en un experimento. Los resultados de estos estudios confirmaron la idea que las personas no son naturalmente hábiles para producir o percibir la aleatoriedad (FALK; KONOLD, 1997; NICKERSON, 2002).

Konold (1991) considera que lo que piensan los estudiantes acerca de la aleatoriedad juega un papel importante en la comprensión de las distribuciones de probabilidad; esto lo llevó a realizar un estudio exploratorio sobre los criterios subjetivos que usan las personas sobre la aleatoriedad; con 20 estudiantes de psicología y 5 de matemática; a quienes se les pidió clasificar los sucesos involucrados en dieciseis ítems, en aleatorios o no aleatorios. Este autor clasifica a los ítems en estocásticos y en reales. Los sucesos de los ítems estocásticos suponen un proceso repetible, como por ejemplo lanzar un dado; mientras que los sucesos de los ítems reales hacen referencia a experiencias de la vida cotidiana, como por ejemplo la germinación de una semilla. El estudio arrojó los siguientes resultados: es mayor el porcentaje de ítems estocásticos, que el porcentaje de ítems reales reconocidos como aleatorios; las argumentaciones de los sujetos en relación a la aleatoriedad ha dado lugar a una amplia gama de concepciones y algunos estudiantes de matemática han expresado su dificultad al tener que dicotomizar los ítems porque ellos tienden a ver el azar como una entidad que se manifiesta en diferentes graduaciones.

Azcárate (1996) realizó un estudio con 57 futuros profesores de Educación Primaria, a quienes les propuso una serie de enunciados en que se describían diferentes fenómenos, y se les preguntaba a los estudiantes si dichos fenómenos eran aleatorios o no y además se les solicitaba que justificaran su respuesta. Los resultados mostraron que pocos sujetos mostraban una idea clara sobre los fenómenos aleatorios; y en la mayoría de los casos fundamentaban desde presupuestos casuales y tuvieron una fuerte influencia los aspectos contextuales. Además, observó una falta de esquemas combinatorios y ausencia de nociones elementales que les permitieran asignar probabilidades a los sucesos y cuantificaban la ocurrencia de los 
fenómenos desde criterios personales. Logra determinar cinco tendencias de pensamiento: Determinista, Causal, Estándar, Incertidumbre e Indeciso.

Lecoutre et al. (2006) continuando con la línea de investigación de Konold (1991), exploraron las creencias subjetivas sobre la aleatoriedad y la probabilidad en tres grupos de estudiantes: un grupo de veinte estudiantes de los últimos años de secundaria, que no habían tenido estadística ni probabilidad; otro grupo de veinte investigadores de psicología, todos con un doctorado y veinte investigadores de matemática, también con un doctorado, a los que se les aplicó un cuestionario con dieciséis ítems; ocho ítems hacían referencia a eventos de la vida cotidiana, en cuatro de los ocho el sujeto estaba implicado en la formulación del ítem, no ocurriendo lo mismo en los otros cuatro; y, finalmente, ocho ítems estocásticos que suponían un proceso repetible; cuatro implicaban dos resultados igualmente probables y los otros cuatro implicaban resultados que no eran equiprobables. De esta forma, pudieron detectar algunas concepciones generales de aleatoriedad. En los ítems reales se observaron dos concepciones principales: por una parte están los que reconocen la aleatoriedad debido a que es posible calcular su probabilidad y otros consideran que el azar no está presente porque el determinismo desempeña un papel importante o bien porque se han identificado factores causales.

Otro resultado importante obtenido por estos autores fue el poco efecto que tuvo el conocimiento de la teoría de la probabilidad sobre la aleatoriedad. Aproximadamente un tercio de los psicólogos clasificaron cada ítem estocástico como no aleatorio, mientras que en los otros dos grupos todos los ítems estocásticos se clasificaron como aleatorios en casi todos los casos. Para algunos psicólogos un ítem no es aleatorio cuando es posible calcular su probabilidad; y clasifican a los ítems reales como no aleatorios con más frecuencia que otros sujetos. Una característica del grupo de los matemáticos es que explicitan dos tipos de aleatoriedades: una matemática, cuando es fácil calcular la probabilidad objetiva, generalmente con los ítems estocásticos; y una aleatoriedad, cuando no es fácil calcular una probabilidad debido a la falta de información respecto del modelo probabilístico estándar, que sería en el caso de los ítems reales.

Serradó, Azcárate y Cardeñoso (2006) realizaron una investigación utilizando cuestionarios y entrevistas para comprender las razones por las cuáles los profesores omiten la enseñanza de la probabilidad en la escuela y las fuentes de información que pueden influir en esta decisión. Realizaron un estudio de casos con cinco profesores; presentaron los resultados obtenidos de dos de ellos, ninguno de los cuales enseñaba probabilidad. El primero (Profesor 1) era licenciado en matemáticas con especialidad en estadística y 5 años de antigüedad en la 
docencia; el segundo (Profesor 2) también era licenciado en matemáticas, pero no era especialista en estadística, con 2 años de experiencia docente. Las fuentes de información consideradas por el profesor 1, fueron el contacto con sus colegas que propiciaban innovaciones en la enseñanza de las matemáticas, pero su omisión en relación con la enseñanza de la probabilidad se debía a su creencia de que la probabilidad no tenía consistencia educativa en la enseñanza obligatoria. Este profesor argumentaba desde una visión formal de la matemática que había adquirido en su formación como profesor, como en los libros de texto de secundaria. El profesor 2 no estaba de acuerdo en enseñar probabilidad pensando en las dificultades que podrían tener los estudiantes, y suponía que la metodología que usaba habitualmente no era válida para este tipo de contenido. A lo que se suma, la falta de fuentes de información que constituía otro obstáculo. A su vez, los docentes manifestaron tener teorías subjetivas sobre los objetivos y contenidos, que hacen que se alejen de las indicaciones dadas en las orientaciones curriculares.

Chernoff (2009) analizó las respuestas de 239 futuros profesores, 163 de primaria y 76 de secundaria. Estos sujetos debían reconocer secuencias formadas por 5 repeticiones del lanzamiento de una moneda. Para el análisis se consideraron las respuestas de 19 sujetos que habían contestado en forma errónea, debido a que consideraron alguna de las siguientes tres interpretaciones del espacio muestral: a) considerando los cambios de cara a cruz; b) considerando la longitud de la racha más largo; c) teniendo en cuenta los cambios de cara a cruz y la longitud de la racha más larga. Por lo tanto los errores incorrectos podrían ser consistentes con estas interpretaciones del espacio muestral; y, en consecuencia, sería por el uso de probabilidades subjetivas personales.

Mohamed y Ortiz (2011) realizaron un estudio de evaluación del razonamiento probabilístico en futuros profesores de primaria. Para ello analizaron las respuestas de 283 estudiantes a dos problemas relacionados con la toma de decisiones, que han sido tomados de Fischbein y Gazit (1984). El estudio indica que existen dificultades en la resolución de los problemas planteados, encontrando factores subjetivos como la heurística de la representatividad, el efecto de la recencia negativa o falacia del jugador y dificultades en la percepción de la independencia de sucesos.

Batanero et al. (2012) analizan las respuestas abiertas a un problema utilizado en las investigaciones sobre percepción subjetiva de la aleatoriedad. El estudio se realizó con 157 futuros profesores de Educación Primaria. Los datos se tomaron como parte de una actividad práctica en la asignatura Enseñanza y Aprendizaje de las matemáticas, después se discutieron las respuestas con los futuros profesores y se realizaron actividades de simulación para 
ayudarles a reconocer las intuiciones erróneas. Los resultados muestran una mezcla de concepciones correctas e incorrectas, algunas de las cuáles son paralelas a las que el concepto de aleatoriedad ha recibido a lo largo de su historia.

En particular, Cardeñoso (2001) realizó su investigación sobre las concepciones probabilísticas en 598 profesores de primaria en activo, y encontró-estabilizó cinco tendencias de pensamiento, a partir de un sistema de nueve categorías: causalidad, multiplicidad, incertidumbre, subjetiva, contingencia, laplaciana, frecuencial, equiprobabilidad y experiencial; que se describen a continuación:

$\checkmark$ Causalidad: Argumentaciones que tienen como criterio de reconocimiento de la aleatoriedad explicaciones en función de los diversos factores causales o en la ausencia de posibilidad de su control.

$\checkmark$ Multiplicidad: Argumentaciones que tienen como criterio de reconocimiento de la aleatoriedad la existencia de múltiples posibilidades en el desarrollo del fenómeno.

$\checkmark$ Incertidumbre: Argumentaciones en las que se utiliza como criterio de reconocimiento de la aleatoriedad la propia imprevisibilidad del suceso, sin profundizar en su explicación o análisis.

$\checkmark$ Subjetiva: Argumentaciones en las que utiliza como criterio de reconocimiento de la aleatoriedad consideraciones referidas a la propia vivencia o creencia subjetiva.

$\checkmark$ Contingencia: Argumentaciones estimativas de cuantificación de la probabilidad basadas en la comparación entre los casos favorables y desfavorables de un suceso.

$\checkmark$ Laplaciana: Argumentaciones estimativas de cuantificación de la probabilidad basadas en la proporción entre los casos favorables y desfavorables del fenómeno.

$\checkmark$ Frecuencial: Argumentaciones estimativas de cuantificación de la probabilidad basadas en la lectura frecuencial del fenómeno o de la información aportada.

$\checkmark$ Equiprobabilidad: Argumentaciones estimativas de cuantificación de la probabilidad basadas en justificaciones desde la equiposibilidad entre los resultados del fenómeno.

$\checkmark$ Experiencial: Argumentaciones estimativas de cuantificación de la probabilidad basadas en criterios fruto de la experiencia personal.

A partir de la aplicación de este sistema de nueve categorías, obtuvo cinco tendencias de pensamiento:

La Concepción Determinista, que niega la existencia del azar, aunque tiene una buena capacidad calculística, lo cual le permite tratar el azar en términos de probabilidades, aunque cuando hace esto, afirma que ya no es azar, porque si hay una regla ya son matemáticas. Cuando no puede responder a la evidencia de la incertidumbre, lo 
califica de excepcional. Como para el determinismo el azar sólo puede ser epistemológico, considera preferibles las teorías científicas de las que se desprenden leyes en las que no tiene cabida el azar.

La Concepción Causal que acepta el azar como excepción a la regla de la ley determinista o en ausencia de información para generar dicha ley científica, usa el azar como variable a controlar, personalmente incluso, afirmando que el saca casi siempre cruz al lanzar una moneda al aire, por ejemplo. Sus explicaciones para las cuestiones imprevisibles están relacionadas con la vieja concepción helena de las cadenas causales. Bajo el punto de vista del cientifismo clásico un evento era aparentemente aleatorio cuando no podía establecerse o controlarse su causa. También se podía asimilar a la ignorancia, como decía Laplace.

> La Concepción Personalista, también como las anteriores de corte pre-indeterministas; aunque el azar y lo indeterminado son cuestiones de carácter mágico o fenomenológico, esotérico, cabalístico, o una cuestión del destino o de casualidades como razón de los sucesos fortuitos.

$>$ La Concepción llamada de Incertidumbre, que reconoce la existencia de lo incierto de la vida, pero se queda prisionero en el proceso de reconocer la existencia de fenómenos azarosos y sucesos aleatorios, como si estuviera luchando para conseguir la conservación de esta atribución de los fenómenos. Utiliza las llamadas intuiciones primarias sobre los fenómenos y sucesos imprevisibles.

$>$ Concepción Contingente, caracteriza a los sujetos que discriminan los fenómenos aleatorios y supeditan lo real a lo posible. No solo busca saber que ocurre, sino también conocer las causas que originan la variación de lo esperado respecto a lo posible.

La aplicación del sistema de categorías propuesto por Cardeñoso (2001) para la determinación de tendencias de pensamiento probabilístico entre los futuros profesores de Biología, no detectó la presencia de las cinco tendencias; que él encontrara entre los maestros andaluces en actividad. En particular, en nuestros estudiantes no aparece el grupo Causalidad; si bien de manera global los estudiantes de Biología usan con mayor frecuencia la causalidad que los maestros andaluces. Por lo que podríamos argumentar, de manera hipotética, que la ausencia de este grupo puede ser debido a la causalidad intrínseca de la Biología; como estructura lógica subyacente a todo el conocimiento biológico de referencia. Por lo tanto, esta formación diferenciada de los estudiantes analizados en este trabajo, respecto de los maestros de primaria; determinan una clasificación que difiere de la tomada como referencia. Por lo 
que nos planteamos la necesidad de seguir investigando el pensamiento probabilístico de los futuros profesores de Biología, para obtener informaciones que nos permitan justificar en profundidad la ausencia del grupo Causalidad.

Por otra parte, tomamos como referencia los aportes dados por Azcárate (1995) en cuanto a la formación del profesorado y el conocimiento profesional del mismo. Esta autora caracteriza el conocimiento profesional como un conocimiento organizado y jerarquizado en complejas tramas que integran distintos núcleos de manera interactiva. Desde esta perspectiva, el aprendizaje profesional se basa en una continúa interacción dialéctica entre las ideas de que dispone para orientar su intervención y las nuevas informaciones y experiencias que le va aportando la realidad en la que interviene, lo que permite la continua reestructuración de sus concepciones de partida y la construcción progresiva de un conocimiento profesional de mayor complejidad.

Desde esta línea adquiere importancia el conocimiento de las concepciones de los estudiantes sobre diferentes elementos del curriculum. En particular, nuestro interés es el conocimiento probabilístico porque proporciona una filosofía del azar, que les permitirá la comprensión del mundo actual y afrontar la toma de decisiones cuando disponga de datos inciertos. Y, como expresa Azcárate (1995), la interpretación probabilística de la realidad conlleva un cambio sustancial en la perspectiva formativa del individuo.

\section{Metodología}

Hemos tomado como base el cuestionario diseñado por Cardeñoso (2001), que aplicara a profesores de educación primaria en actividad; sobre el cual hemos realizado algunas modificaciones lingüísticas, y otras debido a las diferencias en las características físicas y climáticas de nuestro país; como así también hemos tenido en cuenta que los sujetos a los que se les aplicaría el cuestionario son actualmente estudiantes (MORENO; CARDEÑOSO; GONZÁLEZ, 2010).

El cuestionario (CCP_1) se elaboró teniendo en cuenta nueve categorías, y quedando estructurado en dos dimensiones: $A$ y $P$. La dimensión $A$ está definida por los criterios de reconocimiento de la aleatoriedad, y la dimensión $P$ por la argumentación y estimación de la probabilidad. El cuestionario consta de doce ítems correspondientes a la dimensión $A$ y los otros 12 a la dimensión $P$. Estos ítems se encuadran en tres tipologías de contextos: Juego, Cotidiano y Físico Natural; quedando cuatro ítems por contexto para cada una de las dimensiones. En cada uno de los ítems se ha presentado en primer lugar una elección, en el 
caso de la dimensión $A$, el estudiante debe decidir si el suceso presentado es aleatorio o si no es aleatorio; y en el caso de la dimensión $P$, el estudiante elige una de las tres cotas de estimación de la probabilidad en relación con la ocurrencia del suceso presentado: baja, media y alta. Para justificar la elección se les solicita la elección de una opción de respuesta abierta o de una de las tres o cuatro opciones cerradas, que ejemplifican las categorías correspondientes a cada dimensión.

El cuestionario se aplicó a una muestra piloto de 75 estudiantes del Profesorado de Educación Primaria del Instituto de Formación Docente 9-002 de la Ciudad de Mendoza. A partir de las respuestas obtenidas se realizaron nuevas modificaciones, se reemplazaron dos ítems, y a otros se le incorporaron otras opciones. Con estas nuevas modificaciones obtuvimos el cuestionario CCP_2, el que se aplicó a una segunda muestra piloto de 100 estudiantes del Profesorado de Biología del mismo Instituto. Con esta segunda muestra analizamos las características psicométricas del cuestionario: índice de dificultad e índice de discriminación; y la confiabilidad del cuestionario.

A modo de ejemplo, presentamos un ítem correspondiente al reconocimiento de la aleatoriedad y otro ítem de la estimación de la probabilidad.

Ítem 3: "Predecir la cantidad de caras que se obtienen en los 100 lanzamientos de una moneda es un suceso.... ,."

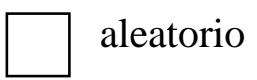
no aleatorio

1. porque ya que puede caer cara o cruz cada vez que se lance, no puedes saber cuántas saldrán. (incertidumbre)

2. porque el número de caras que se obtienen en los 100 lanzamientos puede variar cada vez que lo repitamos. (multiplicidad)

3. porque, para que salga una u otra cara, en cada lanzamiento depende de la posición inicial, de la fuerza con que la tires, del ángulo con que caiga, ... (causalidad)

4. porque.

Ítem 10: "Si en una mesa de juego se dispone de una caja de fichas, que contiene 29 fichas negras y 16 fichas amarillas; la confianza que tengo en que salga una ficha negra, a lo largo de toda una tarde de juego es ........"

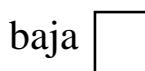

media

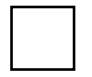

alta

1. porque todas las fichas tienen las mismas posibilidades, en cualquier extracción puede salir tanto una ficha amarilla como una negra. (equiprobable)

2. porque en esta caja hay más fichas negras y puede salir más fácilmente este color. (contingente) 
3. porque hay una proporción de 29 a 45 de fichas negras en la caja. (laplaciana)

4. porque si repetimos la extracción es así de frecuente que esto ocurra. (frecuencial)

5. porque.

La población sobre la que se hace el estudio experimental está constituida por estudiantes que cursan el profesorado de Biología en los institutos de formación docente de la provincia de Mendoza durante el período 2011-2012. El tamaño de la población es de 325 estudiantes. Como nuestro interés es saber si cada uno de los estudiantes reconoce o no la aleatoriedad y desde que categoría justifica su elección; y además qué estimación realizan de la probabilidad y qué argumentos justifican su elección; hemos registrado la frecuencia de cada una de las categorías, por ello resultó apropiado considerar las variables definidas por Cardeñoso (2001), de la forma ALEAij; si i es 1 si indica que el estudiante reconoció la aleatoriedad del suceso, si es 2 indica que no la reconoció y si es 0 que no contestó. La j indica desde que categoría realiza la argumentación; si j=0 indica que no argumenta, si j=1 indica que ha contestado desde la causalidad, si $\mathrm{j}=2$ indica que lo hizo desde la multiplicidad, si $\mathrm{j}=3$ indica la incertidumbre y si $\mathrm{j}=4$ indica la subjetiva. ALEA11, ALEA12, ALEA13, ALEA14, ALEA10, ALEA20, ALEA21, ALEA22, ALEA23, ALEA24. Para la estimación de la probabilidad las variables que representan las frecuencias de las categorías contingencia, laplaciana, equiprobabilidad y experiencial se han representado por PRO5, PRO6, PRO7, PRO8 y PRO9, respectivamente.

La validación del cuestionario se llevó a cabo mediante la aplicación de: índice de dificultad de los ítems, índice discriminante de los ítems, alfa de Cronbach (MORENO; CARDEÑOSO; GONZÁLEZ, 2011).

El índice de dificultad valora la dificultad que implica la resolución del ítem para la población a la que va dirigido el test. El valor del índice varía entre 0 y 1 . El 0 indica una alta dificultad, ya que nadie ha contestado correctamente, y 1 representa la máxima facilidad. Comparando estos índices con los obtenidos por Cardeñoso (2001) comprobamos que ocurre lo mismo respecto de la tipología de ítems con mayor dificultad; siendo estos índices menores en este autor; por ejemplo, para el ítem 7, ítem 8, ítem 9, ítem 11 e ítem 12 (marcados con* en la Tabla 1); los índices de dificultad obtenidos por este autor fueron, respectivamente, 0,$286 ; 0,502 ; 0,314 ; 0,552$ y 0,421 ; lo que nos indica una mayor dificultad para los maestros que para los estudiantes del profesorado de Biología. 
Los índices de discriminación permiten determinar la capacidad del ítem para discriminar los sujetos que poseen una característica de los que no la poseen (en este caso, que reconozcan o no la aleatoriedad de un fenómeno). En nuestro caso un ítem tiene un elevado poder de discriminación si lo aciertan casi todos aquellos estudiantes que han tenido un número importante de aciertos en el cuestionario, y no lo aciertan la mayoría de los que han tenido pocos aciertos en el cuestionario. Hemos calculado este índice en los doce ítems relativos al reconocimiento de la aleatoriedad. Para lo cual, hemos seleccionado dos grupos de estudiantes en función de sus puntuaciones totales de acuerdo el criterio de Kelley (1939), quien mostró que el índice es más sensible cuando se forman dos grupos: uno con el $27 \%$ superior y el otro con el $27 \%$ inferior de los estudiantes, que han sido calificados en función del porcentaje de ítems reconocidos como aleatorios. Una vez identificados los grupos, se calculan las proporciones de aciertos del ítem considerado en los dos grupos, $\mathrm{p}_{\mathrm{s}} \mathrm{y} \mathrm{p}_{\mathrm{i}} \mathrm{y}$ se define el índice de discriminación como: $\mathrm{D}=\mathrm{p}_{\mathrm{s}}-\mathrm{p}_{\mathrm{i}}$ Donde, $\mathrm{p}_{\mathrm{s}}$ es la proporción de estudiantes del grupo superior que responden correctamente al ítem; y donde $\mathrm{p}_{\mathrm{i}}$ es la proporción de estudiantes del grupo inferior que responden correctamente al ítem. El estadístico D varía dentro del intervalo $[-1,+1]$; valores positivos indican que el ítem discrimina a favor del grupo superior, valores negativos indican que el ítem tiene una discriminación invertida. Consideramos el siguiente criterio para la elección de los ítems discriminantes; según Ebel (1965) si: $\mathrm{D} \geq 0,40$ el ítem tiene un funcionamiento satisfactorio; y cuando $\mathrm{D}$ toma valores entre 0,30 y 0,39 el ítem no requiere apenas revisión. Asumiendo este criterio, concluimos que nuestros ítems tienen índices de discriminación aceptables, dado que toman valores entre 0,3703 y 0,7778 .

Tabla 1 - Índices de dificultad y discriminación de los ítems del cuestionario

\begin{tabular}{clccccc}
\multicolumn{4}{c}{ Índices de dificultad } & \multicolumn{4}{c}{ Índices de discriminación } \\
Ítem & contexto & ID & Ítem & $\mathrm{p}_{\mathrm{i}}$ & $\mathrm{p}_{\mathrm{s}}$ & $\mathrm{D}$ \\
\hline 1 & físico natural & 0,73 & 1 & 0,3704 & 0,9629 & 0,5925 \\
2 & juego & 0,78 & 2 & 0,5926 & 1,0000 & 0,4074 \\
3 & cotidiano & 0,80 & 3 & 0,5556 & 1,0000 & 0,4444 \\
4 & juego & 0,90 & 4 & 0,6297 & 1,0000 & 0,3703 \\
5 & cotidiano & 0,82 & 5 & 0,4815 & 1,0000 & 0,5185 \\
6 & juego & 0,83 & 6 & 0,5556 & 1,0000 & 0,4444 \\
7 & cotidiano & $0,51^{*}$ & 7 & 0,2222 & 0,8889 & 0,6668 \\
8 & Cotidiano & $0,60^{*}$ & 8 & 0,2222 & 1,0000 & 0,7778 \\
9 & físico natural & $0,52^{*}$ & 9 & 0,2963 & 0,8148 & 0,5185 \\
10 & juego & 0,80 & 10 & 0,3708 & 1,0000 & 0,6292 \\
11 & físico natural & $0,68^{*}$ & 11 & 0,2222 & 1,0000 & 0,7778 \\
12 & físico natural & $0,65^{*}$ & 12 & 0,2963 & 0,9629 & 0,6666 \\
\hline
\end{tabular}

La confiabilidad de un cuestionario consiste en el grado de relación entre las puntuaciones empíricas y las puntuaciones verdaderas. Es decir, que mide cuán bien el 
cuestionario mide lo que queremos medir. Para comprobar la confiabilidad de los ítems se calculó el coeficiente alpha de Cronbach, obteniéndose el valor 0,749, valor que nos estaría indicando una fiabilidad aceptable.

Los resultados obtenidos en la validación y confiabilidad del cuestionario CCP_2 nos permitieron aplicarlo a toda la población de estudiantes de los Profesorados de Biología de la provincia de Mendoza, integrada por 325 estudiantes.

A las respuestas obtenidas le aplicamos diferentes técnicas estadísticas: análisis de la varianza, test de Tukey, test de independencia, test de Wilcoxon, análisis de clusters y análisis discriminante (MORENO; CARDEÑOSO; GONZÁLEZ, 2012).

El análisis de la varianza univariado se aplicó a la variable ALEAS, que representa la cantidad de sucesos reconocidos correctamente como aleatorios por estudiante, y tomando como factor el contexto del suceso; en nuestro caso el contexto del suceso era de juego, cotidiano y físico-natural. Dado que el resultado que se obtuvo indicó la existencia de diferencias significativas, a continuación se aplicó el test de Tukey para detectar entre qué contextos existen tales diferencias.

El análisis de la varianza multivariado se aplicó a las variables: ALEA11, ALEA12, ALEA13, ALEA14, ALEA21, ALEA22, ALEA23 y ALEA24; que representan la cantidad de estudiantes que reconocen la aleatoriedad por suceso y por categoría; y tomando como factor el contexto del suceso. Como se encontraron diferencias significativas en el reconocimiento de la aleatoriedad en función del contexto, a continuación se aplicó el test de Tukey para determinar qué categorías se aplican con mayor frecuencia en cada contexto. Posteriormente, se aplicó nuevamente el análisis de la varianza multivariado a las variables: PRO5, PRO6, PRO7, PRO8 y PRO9 que representan la cantidad de estudiantes que argumentan la estimación de la probabilidad por cada una de las categorías respectivamente y tomando como factor el contexto del suceso; la obtención de diferencias significativas hizo necesario la aplicación del test de Tukey para determinar qué categorías se aplican con mayor frecuencia en cada contexto.

El test de independencia se aplicó a las variables ALEAS y edad del estudiante; y a las variables ALEAS y nivel académico del estudiante, para determinar si el reconocimiento de la aleatoriedad depende de la edad y del nivel académico de los estudiantes.

El análisis de clusters permitió obtener grupos de estudiantes homogéneos respecto del sistema de categorías propuesto. Posteriormente, la aplicación del análisis discriminante confirmó la clasificación dada por el análisis de clusters y, además, hizo factible la obtención 
de unas funciones discriminantes para clasificar a nuevos estudiantes en alguno de los cuatro grupos, luego de completar el cuestionario.

\section{Resultados}

Las respuestas de los estudiantes al cuestionario fueron analizadas empleando diferentes técnicas estadísticas, que a continuación detallamos.

\subsection{Reconocimiento de la aleatoriedad y el contexto}

El análisis descriptivo aplicado a las respuestas que dieron los estudiantes a los ítems relativos al reconocimiento de la aleatoriedad nos permiten afirmar que el 70,18\% de los estudiantes reconocen la aleatoriedad, resultado similar al encontrado por Cardeñoso (2001), el que registra un $65,5 \%$ de maestros en actividad que reconocen la aleatoriedad, y al de Lecoutre et al. (2006), quienes encontraron que un tercio de los sujetos clasificaron a los ítems estocásticos como no aleatorios.

Tomando como factor el contexto y como variable dependiente la cantidad de estudiantes que reconocen la aleatoriedad en cada ítem, el análisis de la varianza nos indica que el reconocimiento de la aleatoriedad depende del contexto del fenómeno o suceso considerado, con valor $\mathrm{p}=0,046$.

Con la obtención de los intervalos de HSD Tukey para las combinaciones posibles de los tres contextos tomados de a dos obtuvimos los siguientes tres intervalos con una confianza del $95 \%$, respectivamente para contexto de tarea:

$$
\begin{array}{ll}
\text { * Cotidiano_Físico natural } & (-39,30 ; 71,80) \\
\text { * Juego_Cotidiano } & (-14,30 ; 96,80) \\
\text { * Juego_Físico natural } & (1,95 ; 113,05)^{*}
\end{array}
$$

El intervalo $(1,95 ; 113,05)^{*}$ no contiene al cero, lo que informa que existen diferencias significativas entre la cantidad de estudiantes que reconocen la aleatoriedad en los ítems del contexto juego respecto de la cantidad de estudiantes que lo hacen en los ítems del contexto físico-natural. Como afirma Cardeñoso (2001) es esperable que esto ocurra dada la consideración social que tienen los juegos de azar, quien encuentra altamente asociado el contexto de juego con el reconocimiento de la aleatoriedad. Por otra parte, Rouan y Pallascio (1994) plantean la hipótesis de la representatividad del azar asociada al carácter lúdico de la situación, idea que comparten Kahneman y Tversky (1972), pero que en su investigación no 
llegan a confirmar. Sin embargo, Lecoutre et al. (2006) en los ítems que han denominado estocásticos encuentra un mayor reconocimiento de la aleatoriedad en relación con los llamados ítems reales.

\subsection{Las categorías argumentativas de la aleatoriedad}

Las categorías argumentativas más aplicadas son la incertidumbre, con un 40,05\%, seguida por la causalidad, con el 33,13\%, y en tercer lugar la multiplicidad, con un $21 \%$, y, finalmente, la subjetiva, con un 1,59\%. Si comparamos estos resultados con los obtenidos por Cardeñoso (2001) encontramos que este autor detecta un menor uso de la incertidumbre, con un 33\%; un mayor uso de la causalidad, con un 39,2\%; e igual uso de la multiplicidad, con un $20,9 \%$; sin embargo, para la subjetiva su uso es bastante mayor, alcanzando un $5 \%$.

\subsection{Análisis de la varianza aplicado a las categorías argumentativas de la aleatoriedad en función del contexto}

El análisis de la varianza multivariado, tomando como factor el contexto y como variables dependientes la cantidad de estudiantes que eligen cada una de las ocho categorías que argumentan la aleatoriedad, nos informa que existen diferencias significativas en el uso de las categorías ALEA 11, ALEA 13 y ALEA 21 con valores p de 0,033; 0,039 y 0,049, respectivamente. En particular, podemos afirmar que existen diferencias significativas en la aplicación de estas categorías entre el contexto de juego y el físico natural. Los intervalos de Tukey con una confianza del 95\%, aplicados a las combinaciones de las ocho categorías tomadas de a dos nos indican que las categorías entre las cuales existen diferencias significativas son las que se muestran en la Tabla 2.

Concluimos, en forma significativa, que la aleatoriedad se reconoce más, desde la incertidumbre en el contexto de juego y desde la causalidad en el contexto físico natural. Por otra parte, se niega con mayor frecuencia la aleatoriedad en el contexto físico natural que en el contexto de juego desde la causalidad. Lo que confirma los resultados obtenidos por Cardeñoso (2001), quien encuentra que en los ítems correspondientes al contexto físico natural la respuesta mayoritaria, superior al 50\%, es la que se apoya en criterios de causalidad. Por otra parte, Pfannkuch y Brown (1996) afirman que en situaciones del mundo real se aplica el razonamiento causal extraído de la propia experiencia, y Azcárate (1996) argumenta la 
misma idea al afirmar que es producto de la débil comprensión de las ideas básicas probabilísticas.

Tabla 2- Intervalos de confianza de Tukey para detectar diferencias significativas de las categorías de la aleatoriedad en función del contexto

\begin{tabular}{lll}
\hline ALEA 11 & Juego/Físico Natural & $(-108,90 ;-0,60)$ \\
\hline ALEA 13 & Juego/Físico Natural & $(10,24 ; 155,76)$ \\
\hline ALEA 21 & Juego/Físico Natural & $(-114,25 ;-4,75)$
\end{tabular}

\subsection{Relación entre el reconocimiento de la aleatoriedad y las variables nivel académico y edad de los estudiantes}

Consideramos una variable que hemos denominada $A L E A S$, que mide la cantidad de sucesos reconocidos como aleatorios por estudiante; es decir que esta variable toma valores entre 0 y 12 , dado que el estudiante tiene que responder a doce ítems, en cada uno de los cuales se presenta un suceso aleatorio. Además, hemos considerado la variable nivel académico del estudiante. La aplicación del test de independencia a estas variables nos permite afirmar que el reconocimiento de la aleatoriedad es independiente del nivel académico, con un valor de $\chi^{2}=47,782$, g.l. $=36$ y un valor $\mathrm{p}=0,091$. De manera similar, el mismo test aplicado a las variables $A L E A S$ y edad del estudiante, nos proporcionó el mismo resultado, con un valor de $\chi^{2}=50,317$, g.l. $=60$ y valor $\mathrm{p}=0,809$; resultado coincidente con el encontrado por Green (1983) en una muestra de chicos entre 11 y 16 años, quien afirma que el reconocimiento de la aleatoriedad no mejora con la edad; hipótesis que es confirmada por Cardeñoso (2001) en su investigación con maestros en servicio.

\subsection{La estimación de la probabilidad: niveles de confianza y categorías argumentativas}

En la estimación de la probabilidad de los sucesos planteados, la tendencia detectada es la elección del nivel de confianza medio en el 45,05\% de los estudiantes; mientras que el nivel bajo es menos aplicado, sólo es considerado por el 31,54\%; y con menor frecuencia se presenta el alto, con un $17,23 \%$. Resultados que difieren de los encontrados por Cardeñoso (2001), quien registra los porcentajes $41,80 \%, 33,60 \%$ y 23,20\% para los niveles bajo, medio y alto, respectivamente. 
Las argumentaciones que plantean para justificar la elección del nivel de confianza son variadas, dominando la presencia de la categoría equiprobabilidad, con un $31,67 \%$, en segundo lugar se aplica la contingencia, con un $26,79 \%$, seguido por la laplaciana y la frecuencial, con un $17,10 \%$ y $16,51 \%$, respectivamente; mientras que la menos usada es la experiencial, con un 3,36\%. Estos resultados difieren de los encontrados por Cardeñoso (2001), en su estudio encuentra a la contingencia, como la categoría más frecuente, con un $31,3 \%$, seguida de la frecuencial, con un $25,5 \%$, luego la laplaciana, con un 20,5\% y en cuarto lugar la equiprobabilidad, con un 17,3\%; encontramos coincidencias con la experiencial, que igualmente alcanza un valor muy bajo, un $2,9 \%$.

Por otra parte, analizamos la relación entre las categorías argumentativas de la probabilidad y el contexto del suceso; con un valor $\mathrm{p}=0,02198$ encontramos diferencias significativas en el uso de la categoría laplaciana en el contexto físico natural respecto del contexto cotidiano; mediante el intervalo de Tukey obtenido, con una confianza del $95 \%$ es: $(-35,66 ;-3,25)$. Este resultado indica que se usa con menor frecuencia la categoría laplaciana en el contexto físico natural que en el contexto cotidiano.

\subsection{Análisis de Clusters y Análisis Discriminante}

El estudio exploratorio realizado con los 325 estudiantes de los profesorados de Biología de la provincia de Mendoza, Argentina, y cuyos resultados se presentaron en el XVI Simposio de la SEIEM 2012 (MORENO; CARDEÑOSO; GONZÁLEZ, 2012), nos permitió encontrar cinco tendencias de pensamiento probabilístico, que denominamos: Determinista, Causalidad, Contingencia, Incertidumbre y Personalista; Sin embargo, el grupo Personalista quedó integrado por 3 estudiantes. Consideramos que el grupo formado únicamente por tres estudiantes no constituía una tendencia de pensamiento probabilístico representativa de los estudiantes de biología. Por este motivo realizamos un nuevo análisis de clusters que contemplara la clasificación de los estudiantes en cuatro clusters. Para lo cual aplicamos nuevamente el método jerárquico de Ward, que permitió determinar, de manera exploratoria, el número de clusters, mediante el dendograma y la línea de corte (Figura 1); una vez determinado el número de clusters, aplicamos el método de partición de las K-Medias, como sugieren Catena, Ramos y Trujillo (2003).

A partir de la clasificación dada por el análisis de clusters aplicamos el análisis discriminante en SPSS (versión 17), considerando como variables independientes: ALEA 11, ALEA 12, ALEA 13, ALEA 14, ALEA 21, ALEA 22, ALEA 23, ALEA 24, PRO 5, PRO 6 , 
PRO 7, PRO 8 y PRO 9; usando el método inclusión por pasos y considerando que en el cálculo de las probabilidades previas se tuvieran en cuenta los tamaños de los grupos. Los nuevos grupos obtenidos a partir de esta clasificación no difieren mayormente de los obtenidos por el análisis de clusters. En efecto, el análisis discriminante ha funcionado correctamente en el 93,8\% de los casos originales.

Quedando determinadas cuatro tendencias de pensamiento probabilístico: Determinista, Incertidumbre, Contingente y Personalista.

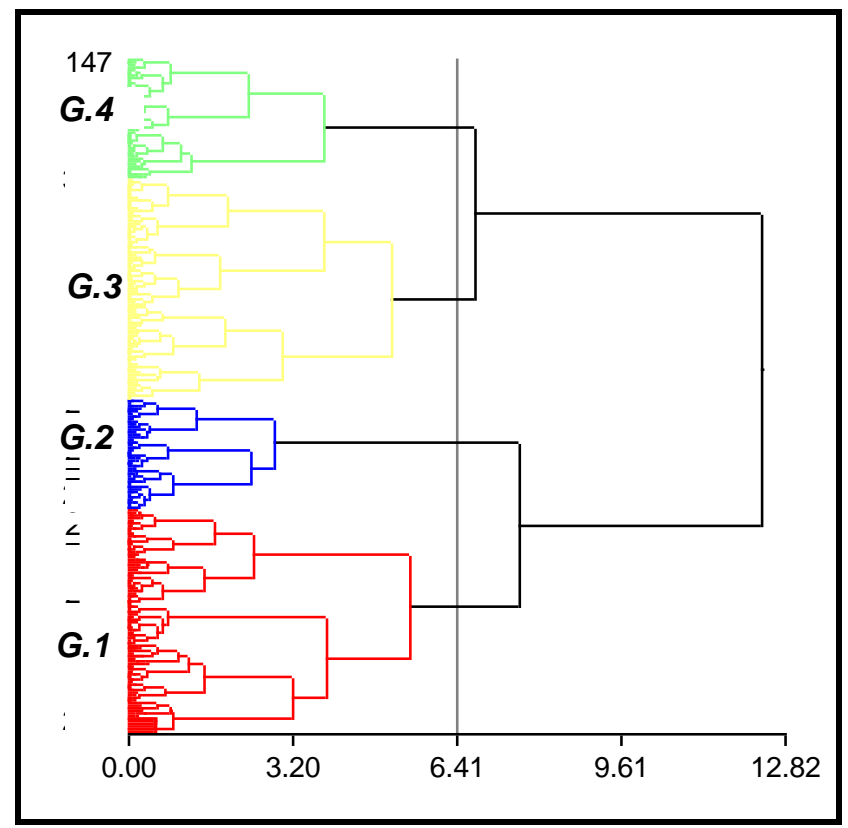

Figura 1- Dendograma del análisis de clusters (G1: Incertidumbre, G2:Determinista, G3:Contingente, G4: Personalista)

Tabla 3 - Clasificación de los estudiantes según el análisis de clusters y del análisis discriminante

\begin{tabular}{ccc}
\hline Grupo & Análisis de clusters & Análisis discriminante \\
Incertidumbre & 128 estudiantes & 133 estudiantes \\
Determinista & 60 estudiantes & 48 estudiantes \\
Contingente & 132 estudiantes & 140 estudiantes \\
Personalista & 5 estudiantes & 4 estudiantes \\
\hline
\end{tabular}

Estos grupos quedan definidos mediante los valores medios que las variables independientes toman en cada uno de ellos. Son precisamente estas medidas las que caracterizan al grupo y determinan la denominación del mismo (Tabla 4). 
Tabla 4 - Valores medios de las trece variables independientes en los 4 grupos

\begin{tabular}{cccccc}
\hline Categorías & Grupo 1 & Grupo 2 & Grupo 3 & Grupo 4 & media \\
\hline ALEA 11 & 2,57 & 1,43 & 2,47 & 3,40 & 2,33 \\
ALEA 12 & 2,14 & 1,22 & 2,11 & 0,40 & 1,93 \\
ALEA 13 & 5,59 & 1,80 & 2,99 & 3,00 & 3,80 \\
ALEA 14 & 0,02 & 0,07 & 0,05 & 1,20 & 0,06 \\
ALEA 21 & 0,91 & 2,25 & 1,99 & 2,00 & 1,61 \\
ALEA 22 & 0,17 & 1,55 & 0,45 & 0,00 & 0,54 \\
ALEA 23 & 0,32 & 3,12 & 0,67 & 0,20 & 0,98 \\
ALEA 24 & 0,09 & 0,07 & 0,14 & 0,60 & 0,12 \\
PRO 5 & 2,96 & 3,03 & 3,70 & 1,40 & 3,25 \\
PRO 6 & 1,43 & 1,68 & 2,86 & 1,00 & 2,05 \\
PRO 7 & 1,91 & 1,43 & 2,35 & 1,20 & 1,99 \\
PRO 8 & 5,34 & 5,30 & 1,83 & 1,00 & 3,84 \\
PRO 9 & 0,24 & 0,17 & 0,20 & 6,20 & 0,30 \\
\hline
\end{tabular}

A continuación describimos los cuatro grupos a partir de los valores medios de las trece categorías.

\section{Grupo 3: Contingencia}

Es el grupo más numeroso, representa al 43,08\% (140) de los estudiantes. Un estudiante de este grupo reconoce como aleatorios, en promedio, aproximadamente entre 7 y 8 ítems de los doce; por lo que ocupa el segundo lugar en el reconocimiento de la aleatoriedad; y cuando lo hace argumenta fundamentalmente desde la multiplicidad (ALEA 12) y desde la causalidad (ALEA 11) con valores que superan a las respectivas medias poblacionales. Pero más que las anteriores argumentaciones, aplica la categoría de incertidumbre (ALEA 13), pese a usarla con un valor inferior a la media. Cuando recurre a la categoría subjetiva (ALEA 14) lo hace con un valor aproximadamente igual a la media. La negación de la aleatoriedad la basa fundamentalmente en la causalidad (ALEA 21) con un valor superior a la media, con lo cual confirma una tendencia determinista, y desde su propia subjetividad (ALEA 24), superando ligeramente a la media.

En la estimación de la probabilidad, se destaca la presencia de la contingencia (PRO 5) con un valor máximo, que ha permitido darle esta denominación, sobre todo entendiendo el uso de la multiplicidad del fenómeno que posee, tan imprescindible para un sujeto que estima la probabilidad desde el establecimiento de las relaciones aditivas entre los casos favorables y desfavorables a la ocurrencia de un suceso. También se destaca el uso de la categoría laplaciana (PRO 6), dado que alcanza el valor máximo en este grupo y la frecuencial (PRO 7) con valores superiores a las medias poblacionales, cuestión esperable pues este tipo de sujeto suele presentar un buen desempeño del cálculo. Se destaca la ausencia de categorías argumentativas desde la experiencial (PRO 9). 
Los estudiantes de este grupo si bien reconocen la aleatoriedad de una cantidad aceptable de fenómenos y argumentan fundamentalmente desde la contingencia, en primer lugar, y desde la laplaciana, en segundo lugar, representan la tendencia de pensamiento probabilístico más avanzada entre las detectadas; sin embargo, no alcanzan el nivel de razonamiento probabilístico esperado.

Un representante de este grupo es el estudiante 91, y responde de la siguiente forma:

Ítem 3: "El número de caras que se obtienen en los 100 lanzamientos de una moneda es un suceso aleatorio porque puede variar cada vez que lo repitamos". Argumenta la aleatoriedad desde la multiplicidad.

Ítem 6: "Tengo un nivel de confianza medio que amanezca frío el 14 de Octubre porque en Octubre hay menos días fríos que templados". Argumenta la estimación de la probabilidad desde la contingencia.

Ítem 10: "Si en una mesa de juego se dispone de una caja de fichas, que contiene 29 fichas negras y 16 fichas amarillas; la confianza que tengo en que salga una ficha negra, a lo largo de toda una tarde de juego es alta porque hay más fichas negras que amarillas". Argumenta la estimación de la probabilidad desde la contingencia.

Ítem 11: "La confianza que tengo en sacar una bola roja de una urna que contiene 5 bolas blancas, 5 rojas y 1 azul, es media porque son 6 bolas en contra de 5 buenas". Argumenta la estimación de la probabilidad desde la contingencia.

\section{Grupo 1: Incertidumbre}

Este es el segundo grupo en importancia numérica, representa al 40,92\% (133) de los estudiantes y es el que logra el mayor reconocimiento de la aleatoriedad, en promedio cada estudiante afirma la aleatoriedad de aproximadamente diez de los doce ítems propuestos. Esta afirmación la hace fundamentalmente basándose en la incertidumbre (ALEA 13), ya que esta variable alcanza el valor máximo en este grupo.

También estos estudiantes argumentan desde la causalidad (ALEA 11) y desde la multiplicidad (ALEA 12) con valores superiores a las medias correspondientes de las variables. Cabe destacar que la afirmación de la aleatoriedad desde la categoría subjetiva (ALEA 14) tiene poca presencia, alcanza el valor mínimo en este grupo. Cuando niegan la aleatoriedad lo hacen desde la causalidad (ALEA 21), la multiplicidad (ALEA 22) y desde la incertidumbre (ALEA23) con valores mínimos en relación con los grupos: determinista y contingencia. Como afirma Cardeñoso (2001), el poco uso de la argumentación multiplicista puede estar denotando a un grupo de sujetos que cree innecesario cualquier otro cálculo, una vez que ha logrado reconocer el carácter incierto de la situación, por lo que lo denomina como indeterministas ingenuos. Cuando debe estimar la probabilidad se basa en la equiprobabilidad (PRO 8), categoría que alcanza el máximo valor en este grupo y que es aplicada en más de la mitad de los ítems, cuestión que es coherente con su caracterización como indeterminista 
incipiente. Mientras que el resto de las argumentaciones se basan en: Contingencia (PRO 5), Laplaciana (PRO 6), Frecuencial (PRO 7) y Experiencial (PRO 9) con valores inferiores a las medias poblacionales. Cuando estima la probabilidad se destaca la presencia dominante de la equiprobabilidad, por el simple hecho de estar en presencia de un fenómeno aleatorio. Esto revela la falta de un conocimiento normativo de la probabilidad.

Un representante de este grupo es la estudiante 136, quien reconoce la aleatoriedad de algunos sucesos de la siguiente forma:

Ítem 3: "Predecir la cantidad de caras que se obtienen en 100 lanzamientos de una moneda es un suceso aleatorio porque puede salir cara o cruz cada vez que se lance la moneda"

Ítem 5: "Sufrir un accidente es un fenómeno aleatorio porque yo no sé cuando puedo sufrir un accidente"

Ítem 7: “Obtener el número 23 en la ruleta de 36 números es un suceso aleatorio porque puede caer en el número 23 o como puede que no caiga"

En relación con la estimación de la probabilidad lo hace de la siguiente forma:

Ítem 10: "Tengo una confianza media en sacar una ficha negra, a lo largo de toda una tarde de juego, de una caja que contiene 29 fichas negras y 16 amarillas, porque puede que salga o que no salga"

En este caso argumenta desde la equiprobabilidad.

Coincidimos con Azcárate (1995) y Cardeñoso (2001) que este grupo se encuentra en buenas condiciones para adentrarse en el estudio matemático de la realidad incierta; y, además, podemos compartir la idea de que estos estudiantes se encuentran en un nivel de conocimiento informal del mundo probabilístico.

\section{Grupo 2: Determinista}

Este grupo está integrado por 14,77\% (48) de los estudiantes es el tercer grupo en importancia numérica. Es el que logra el menor reconocimiento de la aleatoriedad, aproximadamente cada estudiante reconoce cuatro ítems como aleatorios, en promedio. En este grupo encontramos los valores máximos para la negación de la aleatoriedad desde la Causalidad (ALEA 21), la multiplicidad (ALEA 22) y la incertidumbre (ALEA 23), lo que nos lleva a identificarlo como el más determinista de los grupos. Sin embargo, en este sentido son los que menos argumentan desde la subjetividad (ALEA 24), cuestión que suele coincidir con la pericia matemática de los sujetos y su apropiación de los modelos deterministas escolares. Se destaca la presencia de los valores mínimos para las categorías (ALEA 11 y ALEA 12). Un estudiante de este grupo no reconoce la presencia de la aleatoriedad en el mundo real. Ante la presencia de un fenómeno del mundo social, natural o físico busca las causas que lo producen y se resiste a dar explicaciones de tipo indeterminista. Sin embargo, es 
factible que algunos estudiantes sean capaces de discriminar entre fenómenos aleatorios de los no aleatorios, pero son incapaces de identificar cuál es uno y cuál es el otro, dada la cantidad de sucesos nombrados como no aleatorios por algunos estudiantes.

Así, también manifiestan un uso máximo de la equiprobabilidad en la estimación de la probabilidad (PRO 8) y el valor mínimo de la experiencial (PRO 9), siendo la contingencia la única categoría en la estimación de la probabilidad que es ligeramente inferior al valor medio poblacional. Para un estudiante de este grupo la estimación de la probabilidad la argumenta fundamentalmente desde la equiprobabilidad, porque para él el fenómeno simplemente puede ocurrir o no ocurrir, independientemente de que lo considere o no aleatorio. No profundiza en el análisis del fenómeno y no se percata de la complejidad del mismo. Podríamos decir que estamos en presencia de un determinismo causal.

Un representante de este grupo es el estudiante 171, quien ha contestado algunos ítems de la siguiente forma:

Ítem 14: "Que me guste la película que voy a ver el próximo sábado en Cinemak, es un suceso no aleatorio porque leo las críticas antes de comprar la entrada". Argumenta la no aleatoriedad desde la causalidad.

Ítem 20: "Creo que germine una semilla plantada es un fenómeno no aleatorio porque sé las condiciones que tengo que ofrecerle para que germine". Argumenta desde la causalidad.

Ítem 23: "Contraer la gripe el mes que viene es un fenómeno no aleatorio porque sé las cosas que tengo que evitar y aquellas que debo hacer para que no ocurra". Argumenta desde la causalidad.

\section{Grupo 4: Personalista}

Es el grupo más reducido, integrado por cuatro estudiantes, que representan al 1,23\% de la población. Ocupa el segundo lugar en el reconocimiento de la aleatoriedad conjuntamente con el grupo Contingencia. Es decir, cada estudiante reconoce, en promedio, como aleatorios entre siete y ocho ítems. Cuando afirma la aleatoriedad lo hace desde la causalidad (ALEA 11) y desde la categoría subjetiva (ALEA 14) con valores máximos. Cuando niega la aleatoriedad, también lo hace desde la causalidad y desde la subjetividad con valores máximos (ALEA 21, ALEA 24). Las categorías argumentativas de la estimación de la probabilidad alcanzan en este grupo los valores mínimos (PRO 5, PRO 6, PRO 7 y PRO 8), a excepción de la categoría experiencial (PRO 9) que emerge con un valor máximo que sorprende. Esta tendencia se caracteriza por hacer uso de sus creencias y de sus experiencias de vida, que le permiten identificar y justificar la naturaleza incierta de los fenómenos.

Este grupo presenta el mayor uso de la categoría subjetiva tanto para afirmar como para negar la aleatoriedad, y, además, para argumentar la estimación de la probabilidad. 
Dado el reducido número de integrantes de este grupo, entendemos que es una tendencia de pensamiento probabilístico que tiene muy poca presencia entre los estudiantes del profesorado de biología.

Un representante de este grupo es el estudiante 310 , y ha contestado de la siguiente forma:

Ítem 5: Reconoce el suceso como aleatorio y argumenta desde la categoría subjetiva al decir: "Sufrir un accidente es un fenómeno aleatorio porque según mi opinión es el destino de cada uno"

Ítem 9: "La confianza que tengo en conocer una persona famosa el mes que viene, es baja porque no visito los lugares a donde asisten los famosos". Argumenta desde la categoría experiencial.

Para obtener confirmación de las conclusiones obtenidas en relación con la clasificación de los estudiantes en los cuatro grupos mencionados, nos planteamos la necesidad en un futuro inmediato, de entrevistar a cada uno de los estudiantes que mejor represente al grupo de pertenencia.

\section{Discusión y conclusiones}

El máximo reconocimiento de la aleatoriedad corresponde a los ítems relativos al contexto de juego; y el mínimo se presenta en los relativos al contexto físico natural. Esto se debe a la tendencia de los seres humanos a buscar relaciones causa-efecto como intentos de reducir la incertidumbre de los fenómenos desconocidos, afirmando la no aleatoriedad de los mismos y argumentando fundamentalmente desde la causalidad o la subjetividad; lo que expresa una tendencia ubicua del pensamiento humano a buscar explicaciones, para reducir la incertidumbre de los sucesos inciertos, que le permitirán actuar sobre ellos; y que responden al instinto de supervivencia o pensamiento cotidiano, pero no a lo que se puede esperar de un docente. Además, hemos comprobado que existe una fuerte relación positiva entre el reconocimiento de la aleatoriedad y las argumentaciones desde la incertidumbre, y una relación del mismo tipo entre el no reconocimiento de la aleatoriedad y las argumentaciones desde la causalidad.

Globalmente, se entiende que los estudiantes para profesor presentan un aceptable nivel de reconocimiento de la aleatoriedad alcanzando un valor promedio de 8 ítems por estudiante. Sin embargo, hemos detectado la presencia de aproximadamente un $15 \%$ de estudiantes que conforman el grupo determinista, lo que es preocupante ya que este tipo de 
pensamiento no deja espacio para el reconocimiento de la variación y de los fenómenos aleatorios que son tan importantes en biología.

Sería de interés conocer hasta qué punto la visión de estos estudiantes puede estar derivada de la tendencia progresiva a disminuir la importancia del papel del azar en la explicación de los procesos biológicos (LANE, 2009).

En oposición al grupo determinista aparece el grupo incertidumbre, que logra un mayor reconocimiento de la aleatoriedad, representa a menos de la mitad de los estudiantes, con un porcentaje que está por debajo de lo esperado; el que hace un uso elevado de la equiprobabilidad, situación que expresa un indeterminismo incipiente, porque no logra fundamentar sus decisiones desde una perspectiva más compleja; lo que indica que no han alcanzado el nivel cognitivo que les permita interpretar los fundamentos probabilísticos de los fenómenos fortuitos, que son tan necesarios para comprender algunas disciplinas de la biología, como la evolución, la genética y la ecología, en las que el azar juega un rol fundamental (MORENO; GONZÁLEZ, 2013).

Entre el grupo contingente y el grupo determinista, se posicionan los otros dos grupos: incertidumbre y personalista. El grupo contingente es el más numeroso y supera ligeramente al grupo incertidumbre; si bien estima la probabilidad argumentando desde posiciones más cercanas a las formales, no logra un nivel en el reconocimiento de aleatoriedad que le permita utilizar una modelización indeterminista apropiada para la comprensión de los fenómenos aleatorios. En cuanto al grupo personalista, logra el mismo nivel que el grupo contingencia en relación con el reconocimiento de la aleatoriedad, pero difiere de éste en sus argumentaciones, basadas en sus creencias y en sus experiencias.

Esta clasificación de los futuros profesores de Biología en cuatro grupos diferentes en cuanto al nivel de complejidad y en cuanto al número de integrantes, entendemos que tiene su sustento en el hecho de que los biólogos han ido modificando su pensamiento rápidamente en la última década, debido fundamentalmente al gran avance de la genética. Esta modificación se revela, en parte, en las ideas que tienen sobre el azar y la probabilidad.

Los aspectos más débiles de los resultados de esta investigación se encuentran en la estimación de las probabilidades de los sucesos, debido a que basan sus argumentos fundamentalmente, en los conocimientos obtenidos a partir de su propia experiencia, y además, no logran reconocer el espacio muestral asociado a cada suceso. Por lo tanto, no perciben que diez de los doce espacios muestrales no son equiprobables; lo que los lleva a argumentar, en la mayoría de los casos, desde la equiprobabilidad, denotando una falta de conocimiento normativo en relación con el campo del conocimiento probabilístico. 
A partir de estas conclusiones, nos planteamos la necesidad de elaborar un sistemas de estrategias para el desarrollo de procesos formativos, que permitan una preparación profesional adecuada en los estudiantes del profesorado de Biología; como sugieren Cardeñoso y Serradó (2006) la concepción de la enseñanza debe estar basada en modelos investigativos, que necesitan de procesos de formación inclusivos y favorezcan la reflexión desde todas las perspectivas sobre el significado del aprendizaje en el campo estocástico. Por lo tanto, debemos tener en cuenta algunas propuestas de investigadores que han logrado resultados satisfactorios; como el realizado por Cazorla, Gusmão y Kataoka (2011). Quienes llevaron a cabo la validación de una secuencia didáctica para la enseñanza de conceptos de probabilidad en la escuela básica, con veintiocho profesores, afirmando las ideas de Batanero y Godino (2002) en relación con el desarrollo del razonamiento probabilístico, desde el enfoque Ontosemiótico de la cognición e instrucción matemática (GODINO; BATANERO; FONT, 2007, 2008).

Hemos de considerar que una adecuada enseñanza de los conceptos que nos ocupan en el profesorado de biología tiene que tener en cuenta no sólo su formación matemática previa, sino también la visión y formación biológica de estos estudiantes. En qué medida esta formación disciplinar puede interactuar con su pensamiento probabilístico es un reto que se nos plantea en el horizonte.

Finalmente, queremos destacar que en el presente trabajo realizado sobre el pensamiento probabilístico de los profesores de Biología en formación, es un tema que no ha sido investigado con anterioridad.

Además, subrayamos dos ideas que consideramos de importancia. En primer lugar, hemos detectado diferencias significativas en el reconocimiento de la aleatoriedad en función del contexto del suceso considerado, siendo mayor en el contexto de juego que en el físiconatural. Mientras que, en el contexto físico-natural se afirma la aleatoriedad desde la causalidad, en el de juego se afirma desde la incertidumbre. Sin embargo, esperábamos que estos futuros profesores de Biología lograran un mayor dominio del contexto físico-natural, dada la especificidad de su formación.

En segundo lugar, no se ha encontrado relación de dependencia entre el reconocimiento de la aleatoriedad y la edad de los estudiantes, como así tampoco con el nivel académico de los mismos. Este resultado sugiere la idea de que el actual diseño curricular de la carrera no ha favorecido la formación probabilística de los estudiantes, dado que la misma no depende del curso en el que se encuentre el estudiante. Por otra parte, se constata que la vida y la experiencia cotidiana no forman el pensamiento, en general, en este campo, y en 
particular, no mejora la capacitación de los estudiantes para reconocer fenómenos o sucesos aleatorios.

\section{Referencias}

AZCÁRATE, P. El Conocimiento Profesional de los Profesores sobre las nociones de Aleatoriedad y Probabilidad. Su estudio en el caso de la educación Primaria. 1995, 957. Tesis (Doctorado en Filosofía y Ciencias de la Educación: Ciencias de la Educación) - Facultad de Educación, Universidad de Cádiz, Cádiz, España, 1995.

AZCÁRATE, P. Estudio de las concepciones disciplinares de futuros profesores de primaria en torno a las nociones de aleatoriedad y probabilidad. Granada: Editorial Comares, 1996.

AZCÁRATE, P.; CARDEÑOSO, J.M. La Enseñanza de la Estadística a través de Escenarios: implicación en el desarrollo profesional. Bolema, Rio Claro, v. 24, n. 40, p.789-810, Dic. 2011. Disponible en:

<http://www.periodicos.rc.biblioteca.unesp.br/index.php/bolema/article/view/5294/4171>. Acceso en: 13 Marzo 2013.

BATANERO, C.; GODINO, J. Didáctica de la estadística y probabilidad para maestros: Probabilidad. En: GODINO, J. (Ed.). Didáctica de las Matemáticas para Maestros. Proyecto Edumat maestros. Granada: Universidad de Granada, 2002, p.425-444. Disponible en:

http://www.ugr.es/ jgodino/edumat-maestros. Acceso en: 18 Marzo 2013.

BATANERO, C.; GÓMEZ, E.; SERRANO, L.; CONTRERAS, J.M. Comprensión de la Aleatoriedad por Futuros Profesores de Primaria. Redimat. Revista de Investigación en Didáctica de las Matemáticas, Barcelona, v.1, n.3, p.222-245, Oct. 2012.

BOROVNIK, M. A plea for a relatively strong role for probability within stochastic curricula. In: BATANERO C.; BURRILL G.; READING C.; ROSSMAN A. (Ed.). Joint ICMI/IASE Study: Teaching Statistics in School Mathematics. 2008. Disponible en:

<www.stat.auckland.ac.nz/ iase/publications/rt08/Panel2_Borovcnik.pdf>. Acceso en 10 de Enero de 2013.

BOROVNICK, M. Strengthening the Role of Probability Within Statistics. In: BATANERO, C.; BURRILL, G.; READING, C. (Ed.). Teaching Statistics in School Mathematics-Challenges for Teaching and Teacher Education. New York: Springer, 2011, p.71-84.

BOROVCNIK, M.; PEARD, R. Probability. In: BISHOP, K.; CLEMENTS, C.; KEITEL, J.; KILPATRICK, C.; LA-BORDE (Ed.). International Handbook of Mathematics Education. Dordrecht: Kluwer, 1996. p. 239-288.

CARDEÑOSO, J. M. Las creencias y conocimientos de los profesores de primaria andaluces sobre la Matemática escolar. Modelización de conceptos sobre la Aleatoriedad y Probabilidad. 2001, 505. Tesis (Doctorado en Filosofía y Ciencias de la Educación: Ciencias de la Educación) Facultad de Educación, Universidad de Cádiz, Cádiz, España, 2001.

CARDEÑOSO, J. M.; SERRADÓ, A. ¿Puedo adivinar qué idioma está hablando mi amigo con sólo contar las vocales? Escenarios para el aprendizaje de la Estadística y la Probabilidad. In: FLORES P.; POZUELO R.; ROA R., (Ed.). Investigación en el aula de matemáticas. Estadística y azar. Granada: Sociedad S.A.E.M. Thales y Universidad de Granada, p.279-301, 2006. Disponible en: 
<http://earlystatistics.net/template/pdf/Cardenoso_serrado_Thales2006_taller.pdf $>$. Acceso en: 14 Marzo 2013.

CATENA, A.; RAMOS, M.; TRUJILlO, H. M. Análisis Multivariado. Un manual para investigadores. Madrid: Ed. Biblioteca Nueva, 2003.

CAZORLA, I.; GUSMÃO, T.C.; KATAOKA, V.Y. Validação de uma Sequência Didática de Probabilidade a partir da Análise da Prática de Professores, sob a Ótica do Enfoque Ontossemiótico. Bolema, Rio Claro, v. 24, n. 39, p. 515-536, ago. 2011. Disponible en:

<http://www.periodicos.rc.biblioteca.unesp.br/index.php/bolema/article/view/5107>.. Acceso en: 8 Marzo 2013.

CHERNOFF, E. Subjetive probabilities derived from the perceived randomness of sequences of outcomes. 2009, 119. Tesis (Doctorado Educación)-Simon Fraser University, Vancouver, Canada, 2009.

EBEL, R.L. Measuring educational achievement. Englewood Cliffs: Prentice Hall, 1965.

FALK, R.; KONOLD, C. Making sense of randomness: implicit encoding as a basis for judgment. Psychological Review, Washington, v. 104, n. 2, p. 301-318. abril 1997.

FISCHBEIN, E.; GAZIT, A. Does the teaching of probability improve probabilistic intuitions? Educational Studies in Mathematics, New York, v.15, n.1, p.1-24, febrero 1984.

GODINO, J.D.; BATANERO, C.; FONT, V.T. The onto-semiotic approach to research. In: mathematics education. ZDM. The International Journal on Mathematics Education, New York: Springer, v. 39, n. 1, p. 27-135, enero. 2007.

GODINO, J. D.; BATANERO, C.; FONT, V. Um enfoque onto-semiótico do conhecimento e a instrução matemática. Acta Scientiae, Revista de Ensino de Ciências e Matemática, Canoas, v.10, n.2, p.7-37, diciembre 2008.

GREEN, D.R. A survey of probabilistic concepts in 3000 pupils aged 11-16 years. In: GREY, D.R et al. (Ed.). Proceedings of the First International Conference on Teaching Statistics Trust, 9-13 August 1982. Sheffield: University of Sheffield volumen 2, p.766-783, junio 1983.

KAHNEMAN, D.; TVERSKY, A. Subjetive Probability: a judgment of representativeness. Cognitive Psychology, United States, v. 3, n. 3, p. 430-454, octubre 1972.

KELLEY, T.L. Selection of upper and lower groups for the validation of test items. Journal of Educational Psychology, Washington, v. 30, p. 17-24, enero 1939.

KONOLD, C. Understanding student's beliefs about probability. In: VON GLASERSFELD, E. (Ed.). Radical Constructivism in Mathematics Education, Dordrecht, 1991. p.139-156.

LANE, N. Los diez grandes inventos de la evolución. Madrid: Editorial Ariel, 2009

LECOUTRE, M.P.; ROVIRA, K.; LECOUTRE, B.; POITEVINEAU, J. People's Intuitions about randomness and probability: an empirical study. Statistics Education Research Journal, Alexandria, v. 5, n. 1, p. 20-35, mayo 2006. Disponible en: 〈http://www.stat.aucland.ac.nz/serj >. Acceso en: 13 Marzo 2013.

MOHAMED, N.; ORTIZ, J. J. Creencias sobre la aleatoriedad de futuros profesores de educación primaria. In: MORENO M. M.; CLIMENT N. (Ed.). Investigación en Educación Matemática. 
Comunicaciones de los grupos de Investigación de la SEIEM, Lleida: SEIEM y Univ. Lleida, 2012.p. 253-265.

MORENO, A.; GONZÁLEZ, F. El concepto de población: Un análisis en el contexto del profesorado de Biología. Saarbrücken: Editorial académica española, 2013.

MORENO, A; CARDEÑOSO, J.M.; GONZÁLEZ, F. Necesidad de conocer los sistemas de ideas probabilísticas de los futuros profesores: Elaborando un cuestionario. In: ENCUENTRO LATINOAMERICANO DE PROFESORES Y ESTUDIANTES DE MATEMÁTICA Y CIENCIAS NATURALES, $1^{\text {th }}$, 2010, Mendoza, Argentina, Anales... Mendoza, Argentina: María del Carmen Navarro y Fabio Lorenzo, 2010. p.21-29 CD-ROM.

MORENO, A.; CARDEÑOSO, J.M.; GONZÁLEZ, F. Las dificultades detectadas en un grupo de estudiantes del profesorado de educación primaria cuando afrontan la asignación de probabilidades. In: SIMPOSIO DE LA SOCIEDAD ESPAÑOLA DE INVESTIGACIÓN EN EDUCACIÓN MATEMÁTICA, 15 ${ }^{\text {th }}$ 2011, Ciudad Real, Anales...Ciudad Real: Universidad de Castilla, La Mancha. Facultad de Educación, Margarita Marín Rodriguez y Nuria Climent Rodriguez, 2012. p.153178. CD-ROM.

MORENO, A.; CARDEÑOSO, J.M.; GONZÁLEZ, F. Un estudio exploratorio de las tendencias de pensamiento probabilístico de los estudiantes del profesorado de biología. In: SIMPOSIO DE LA SOCIEDAD ESPAÑOLA DE INVESTIGACIÓN EN EDUCACIÓN MATEMÁTICA, $16^{\text {th }}, 2012$, Baeza. Anales...Baeza: Antonio Estepa Castro, Ángel Contreras de la Fuente, Jordi Deulofeu Piquet, M. C. Penalva Martínez, F. J. García García, L. Ordóñez Cañada (Eds.), 2012, p.407-416.

MORENO, A.; CARDEÑOSO, J.M.; GÓNZÁLEZ-GARCÍA, F. La aleatoriedad en profesores de biología y matemáticas en formación: análisis y contraste de significados. Revista Eureka sobre Enseñanza y Divulgación de las Ciencias (REurEdC) 11(2), p. 198-215, abril 2014 Disponible en:

<http://rodin.uca.es/xmlui/bitstream/handle/10498/15975/06-583-Moreno.pdf?sequence=6>.

Acceso el 3/5/2014

PFANNKUCH, M.; BROWN, C.M. Building on and Challenging Students'Intuition About Probability: Can We Improve Undergraduate Learning? Journal of Statistics Education, Auckland, v. 4, n. 1, 1996. Disponible en: <http://www.amstat.org/publications/jse/v4n1/pfannkuch.html>. Acceso en: 14 mar. 2013.

ROUAN, O.; PALLASCIO, R. Conceptions probabilistes d'éléves marocains du secondaire. Recherches en Didactique des Mathématiques, Paris, v. 14, n. 3, p. 393-428, octubre 1994.

RUIZ, B.; BATANERO, C.; ARTEAGA, P. Vinculación de la Variable Aleatoria y Estadística en la Realización de Inferencias Informales por parte de Futuros Profesores. Bolema, Río Claro, v. 24, n. 39, p.431-449, Ago. 2011. Disponible en:

<http://www.periodicos.rc.biblioteca.unesp.br/index.php/bolema/article/view/5102>. Acceso en: 16 mar. 2013.

SERRADÓ, A.; AZCÁRATE, P.; CARDEÑOSO, J.M. Analyzing teacher resistance to teaching probability in compulsory education. In: THE INTERNATIONAL CONFERENCE ON TEACHING STATISTICS, 7, 2006, Salvador de Bahía. Proceedings... Salvador de Bahía: International Association for Statistical Education, 2006, p.1-20 (ROSSMAN, A. y CHANCE, B. (Eds.). Disponible en:< http://www.ime.usp.br/ abe/ICOTS7/Proceedings/PDFs/InvitedPapers/2E4_SERR.pdf>. Acceso en:

10 Julio 2012. 
WAGENAAR, W.A. Generation of random sequences by human subjects: A critical survey of the literature. Psychological Bulletin, Washington, v. 77, n. 1, p. 65-72, jan. 1972.

Submetido em Junho de 2013. Aprovado em Agosto de 2013. 